\title{
Navigation Performance Enhancement Using IMM Filtering for Time Varying Satellite Signal Quality
}

\author{
Dah-Jing Jwo *, Shu-Ming Chang and Jen-Hsien Lai \\ Department of Communications, Navigation and Control Engineering, National Taiwan Ocean University \\ 2 Pei-Ning Rd., Keelung 202, Taiwan
}

\begin{abstract}
A novel scheme using fuzzy logic based interacting multiple model (IMM) unscented Kalman filter (UKF) is employed in which the Fuzzy Logic Adaptive System (FLAS) is utilized to address uncertainty of measurement noise, especially for the outlier types of multipath errors for the Global Positioning System (GPS) navigation processing. Multipath is known to be one of the dominant error sources, and multipath mitigation is crucial for improvement of the positioning accuracy. It is not an easy task to establish precise statistical characteristics of measurement noise in practical engineering applications. Based on the filter structural adaptation, the IMM nonlinear filtering provides an alternative for designing the adaptive filter in the GPS navigation processing for time varying satellite signal quality. The uncertainty of the noise can be described by a set of switching models using the multiple model estimation. An UKF employs a set of sigma points by deterministic sampling, which avoids the error caused by linearization as in an extended Kalman filter (EKF). For enhancing further system flexibility, the fuzzy logic system is introduced. The use of IMM with FLAS enables tuning of appropriate values for the measurement noise covariance so as to obtain improved estimation accuracy. Performance assessment will be carried out to show the effectiveness of the proposed approach for positioning improvement in GPS navigation processing.
\end{abstract}

\section{Introduction}

Multipath [1-4] is known to be one of the dominant error sources in high accuracy global navigation satellite systems (GNSS) positioning systems, such as the Global Positioning System (GPS)[5]. Since multipath errors are among uncorrelated errors that are not cancelled out during observation differencing, the performance of high precision GPS receivers are mostly limited by the multipath induced errors. Most of the existing multipath mitigation algorithms are designed for Gaussian noise. However, non-Gaussian noise is often encountered in many practical environments and their performances degrade dramatically in non-Gaussian cases. The extended Kalman filter (EKF) [5] has been shown to be a minimum mean square error estimator. Unfortunately, the fact that EKF highly depends on a predefined dynamics model forms a major drawback. For achieving good filtering results, the designers are required to have the complete a priori knowledge on both the dynamic process and measurement models, in addition to the assumption that both the process and measurement are corrupted by zero-mean Gaussian white sequences. Moreover, the calculation of the Jacobian matrices makes it difficult to implement, especially for the nondifferentiable function.

The nonlinear filter is designed to give a preliminary estimation of the state. The mean square error criterion is limited to the assumption of linearity and Gaussianity. Multipath effects occur when GPS signals arrive at a receiver site via multiple paths due to reflections from nearby objects, such as the ground and water surfaces, buildings, vehicles, hills, trees, etc. Many multipath estimation algorithms have been studied to eliminate the positioning error caused by multipath. Although the particle filter (PF) [3] can deal with nonGaussian, nonlinear system, it is computationally expensive for an online realization. The nonlinear filters considered in this research include the unscented Kalman filter (UKF) [7,8]. The UKF naturally suffers the same problem as the EKF. The uncertainty of the process noise and measurement noise will degrade the performance of UKF.

The adaptive Kalman filter [9,10] algorithm has been one of the strategies considered for estimating the state vector to prevent divergence problem due to modeling errors. An adaptive mechanism which dynamically identifies uncertainties or modeling errors can be adopted. To deal with the noise uncertainty and system nonlinearity simultaneously, the IMMUKF can be introduced. In the approach, these multiple models are developed to describe various dynamic behaviors. In each model an UKF is running, and the IMM algorithm makes uses of model probabilities to weight the inputs and output of a bank of parallel filters at each time instant.

\footnotetext{
* Corresponding author: djjwo@ mail.ntou.edu.tw
} 
The interacting multiple model (IMM) [11-14] algorithm has the configuration that runs in parallel several model-matched state estimation filters, which exchange information (interact) at each sampling time. The IMM algorithm has been originally applied to target tracking, and recently extended to navigation application. A model probability evaluator calculates the current probability of the vehicle being in each of the possible modes. A global estimate of the vehicle's state is computed using the latest mode probabilities. This algorithm carries out a soft-switching between the various modes by adjusting the probabilities of each mode, which are used as weightings in the combined global state estimate. The covariance matrix associated with this combined estimate takes into account the covariances of the mode-conditioned estimates as well as the differences between these estimates.

A fuzzy logic adaptive system (FLAS) $[9,15]$ is added to the UKF innovation term to compensate for the non-Gaussianity of the whole system. The fuzzy logic reasoning system is based on the Mamdani type model. The fuzzy reasoning system is constructed for obtaining the suitable process noise according to the time-varying change in dynamics. By monitoring the innovation information, the FLAS is employed for dynamically adjusting the process noise based on the fuzzy rules so as to enhance the estimation accuracy and tracking capability. The FLAS approach is based on parametric adaptation, while the IMM approach is based on filter structural adaptation (model switching). The example on the GPS navigation processing based on the FLAS based adaptive IMMUKF (Fuzzy-IMMUKF) will be presented. The performance comparison will be demonstrated by using the proposed fuzzy-IMMUKF method as compared to the relatively conventional UKF and IMMUKF approaches.

The remaining of this paper is organized as follows. In Section 2, preliminary background on the multipath interference is reviewed. The interacting multiple model unscented Kalman filter is introduced in Section 3. Section 4 presents the fuzzy adaptive filter strategy. In Section 5, simulation experiments are carried out to evaluate the performance using the proposed fuzzyIMMUKF as compared to the UKF and IMMUKF approaches. Conclusions are given in Section 6.

\section{Multipath interference}

Multipath [1-4] is the replica of direct signal caused by the reflection of buildings, hills and other obstacles. Multipath interference and non-line-of-sight (NLOS) reception are major sources of error for GNSS in an urban environment. It is one of the dominant error sources for high accuracy positioning systems due to the irrelevancy between different instants and the occurrence uncertainty along the observation period. In severe multipath environments, it may be possible that the LOS signal is obstructed completely and only the reflected signals are present.

Multipath signals are always delayed compared to line-of-sight signals because of the longer travel paths caused by the reflection. These multipath effects on the code phase measurements are most crucial, and the multipath error can reach up to a few tens of meters, or a couple of hundred. Unlike other error sources, multipath cannot be mitigated through differential processing, since it decorrelates spatially rapidly. The pseudorange errors caused by the multipath interference can be reduced significantly through carefully designed antenna, such as antenna siting, choke-ring antenna design. Receiver-based multipath mitigation techniques use modified discriminator designs, often with additional correlators, to improve the resolution of the signal correlation process.

The EKF is a useful algorithm for multipath estimation in Gaussian noises. However, it cannot be effectively used to cope with multipath estimation problem with non-Gaussian noises. The particle filter (PF) algorithm has been applied for multipath estimation in non-Gaussian noise environment. PF methods use the sequential importance sampling to characterize the posterior probability density function of multipath parameters. It involves the approximation of the posterior density function by a set of random samples taken from an importance density function. The selection of the density function is critical for PF's performance and the optimal one is usually hard to find in most cases. Furthermore, the problem of particle degeneracy also affects the PF's application in multipath estimation.

The unscented Kalman filter (UKF) made a Gaussian approximation with a limited number of points (sigma points) by using the UT. The deterministic sampling approach is used to capture the mean and covariance estimates with a minimal set of samples, and the posterior mean and covariance undergoing a nonlinear propagation can be calculated accurately at least to the second order.

\section{The interacting multiple model unscented Kalman filter}

The unscented Kalman filtering process model and measurement model are represented as :

$$
\begin{aligned}
\mathbf{x}_{k+1} & =f\left(\mathbf{x}_{k}\right)+\mathbf{w}_{k} \\
\mathbf{z}_{k} & =h\left(\mathbf{x}_{k}\right)+\mathrm{v}_{k}
\end{aligned}
$$

where the state vector $\mathbf{x}_{k} \in \mathfrak{R}^{n}$, process noise vector $\mathrm{W}_{k} \in R^{n}$,measurement vector $\mathbf{z}_{k} \in \mathfrak{R}^{m}$, and measurement noise vector $\mathbf{v}_{k} \in \mathfrak{R}^{m}$. The vectors $\mathbf{W}_{k}$ and $\mathbf{V}_{k}$ are zero mean Gaussian white sequences having zero cross-correlation with each other :

$$
\begin{aligned}
& \mathbf{E}\left[\mathbf{w}_{k} \mathbf{w}_{i}{ }^{\mathrm{T}}\right]=\left\{\begin{array}{ll}
\mathbf{Q}_{k}, & i=k \\
0, & i \neq k
\end{array} ; \quad \mathbf{E}\left[\mathbf{v}_{k} \mathbf{v}_{i}{ }^{\mathrm{T}}\right]=\left\{\begin{array}{ll}
\mathbf{R}_{k}, & i=k \\
0, & i \neq k
\end{array} ;\right.\right. \\
& \mathbf{E}\left[\mathbf{w}_{k} \mathbf{v}_{i}^{\mathrm{T}}\right]=\mathbf{0} \quad \text { for all } i \text { and } k
\end{aligned}
$$

where $\mathbf{Q}_{k}$ is the process noise covariance matrix, $\mathbf{R}_{k}$ is the measurement noise covariance matrix, $E[\cdot]$ represents expectation, and superscript " $\mathrm{T}$ ", denotes matrix transpose.

The IMM algorithm uses two or more models to describe the possible states in the working process. Let a 
general system for multiple models in discrete time be described by:

$$
\begin{aligned}
& \mathrm{x}_{1}(k+1)=f\left(\mathrm{x}_{1}(k)\right)+\mathrm{w}_{1}(k) \\
& \mathrm{x}_{2}(k+1)=f\left(\mathrm{x}_{2}(k)\right)+\mathrm{w}_{2}(k)
\end{aligned}
$$$$
\mathrm{N}
$$

$$
\mathrm{x}_{j}(k+1)=f\left(\mathrm{x}_{j}(k)\right)+\mathrm{w}_{j}(k)
$$

Estimation of the system state is performed by effective weighted fusion, and the state variable (e.g., position, speed, etc.) can be corrected in real time. The adaptability can well overcome the problem of large single-model estimation error.

The unscented Kalman filter (UKF) is a nonlinear and distribution approximation method, which uses a finite number of sigma points to propagate the probability of state distribution through the nonlinear dynamics of system. Unscented Transform (UT) is the basic core of UKF method, which is a method to calculate the statistical characteristics of random variables after nonlinear transformation. UT transformation is based on prior information, and it is easier to approximate the probability density distribution of nonlinear function than to approximate its function. On the premise that the sampling mean and covariance are ensured, a set of Sigma points are selected. The nonlinear transformation is applied to each Sigma point in the sample, and the nonlinear transformation points are obtained by substituting into the nonlinear function.

The IMMUKF algorithm uses model probabilities to weight the input and output of parallel UKF at each time instant. The approach takes into account a set of models to represent the system behavior patterns or system model. The overall estimates is obtained by a combination of the estimates from the filters running in parallel based on the individual models that match the system modes. In each cycle, four major steps are involved: interaction, filtering, mode probability calculation, and combination[15].

\subsection{Model interaction}

For given states $\mathbf{x}_{k-1}^{j}=\mathbf{x}_{k-1 \mid k-1}^{j}$ with corresponding covariances $\mathbf{P}_{k-1}^{j}=\mathbf{P}_{k-1 \mid k-1}^{j}$ and mixing probabilities $\boldsymbol{\mu}_{k-1 \mid k-1}^{i \mid j}$ for each model, , the initial condition for model $j$ is:

$$
\hat{\mathbf{x}}_{k-1 \mid k-1}^{0 j}=\sum_{i=1}^{r} \hat{\mathbf{x}}_{k-1 \mid k-1}^{i} \boldsymbol{\mu}_{k-1 \mid k-1}^{i \mid j}, \quad j=1,2, \ldots, r
$$

with the covariance corresponding to the above is:

$$
\mathbf{P}_{k-1 \mid k-1}^{0 j}=\sum_{i=1}^{r} \boldsymbol{\mu}_{k-1 \mid k-1}^{i \mid j}\left\{\mathbf{P}_{k-1 \mid k-1}^{i}+\left[\hat{\mathbf{x}}_{k-1 \mid k-1}^{i}-\hat{\mathbf{x}}_{k-1 \mid k-1}^{0 j}\right]\left[\hat{\mathbf{x}}_{k-1 \mid k-1}^{i}-\hat{\mathbf{x}}_{k-1 \mid k-1}^{0 j}\right]^{T}\right\}
$$

The model transition probabilities are related to Markov chain and are defined as:

$$
\pi_{i j}=p\left\{M_{k}^{j} \mid M_{k-1}^{i}\right\}=\left[\begin{array}{cccc}
\pi_{11} & \pi_{12} & \mathrm{~K} & \pi_{1 j} \\
\pi_{21} & \pi_{22} & \mathrm{~K} & \pi_{2 j} \\
\mathrm{M} & \mathrm{M} & \mathrm{O} & \mathrm{M} \\
\pi_{i 1} & \pi_{i 2} & \mathrm{~K} & \pi_{i j}
\end{array}\right]
$$

where $i, j=1,2, \ldots, r$, and $r$ is the number of submodels. Calculating the mixing probabilities with mode switching probability matrix $\pi_{i j}$ and the Gaussian mixing probabilities are computed via the equations:

$$
\boldsymbol{\mu}_{k-1 \mid k-1}^{i j}=\frac{1}{\overline{\mathbf{c}}_{j}} \pi_{i j} \boldsymbol{\mu}_{k-1}^{i}
$$

where $\overline{\mathbf{c}}_{j}$ is a normalization factor:

$$
\overline{\mathbf{c}}_{j}=\sum_{i=1}^{r} \pi_{i j} \boldsymbol{\mu}_{k-1}^{i}
$$

\subsection{Model filtering using UKF}

- Stage 1 in UKF loop. The unscented transform creates $2 n+1$ sigma vectors $\mathbf{X}$ and weighted points $W$. For state estimation at instant $k-1$, sigma points are generated according to

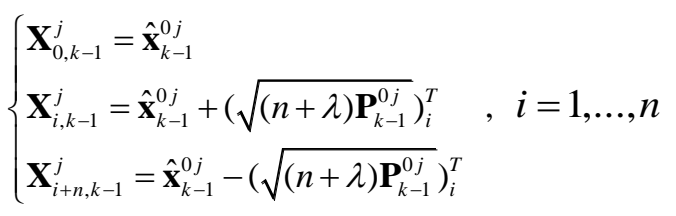

where $\left(\sqrt{(n+\lambda) \mathbf{P}_{k-1}^{0 j}}\right)_{i}$ is the $i$ th row (or column) of the matrix square root. $\sqrt{(n+\lambda) \mathbf{P}_{k-1}^{0 j}}$ can be obtained from the lower-triangular matrix of the Cholesky factorization; $\lambda=\alpha^{2}(n+k)-n$ is a scaling parameter for controling the covariance matrix; $\alpha$ determines the spread of the sigma points and is usually set to a small positive (e.g., $1 e-4 \leq \alpha \leq 1$ ); $k$ is a secondly scaling parameter (usually set as 0 ); $\beta$ is used to incorporate prior knowledge of the distribution of $\overline{\mathbf{x}}$ (When $\mathbf{x}$ is normally distributed, $\beta=2$ is an optimal value); $W_{i}^{(m)}$ is the weight for the mean associated with the $i$ th point; and $W_{i}^{(c)}$ is the weigh for the covariance associated with the $i$ th point, given by

$$
\begin{gathered}
W_{0}^{(m)}=\frac{\lambda}{(n+\lambda)} \\
W_{0}^{(c)}=W_{0}^{(m)}+\left(1-\alpha^{2}+\beta\right) \\
W_{i}^{(m)}=W_{i}^{(c)}=\frac{1}{2(n+\lambda)}, \quad i=1, \ldots, 2 n
\end{gathered}
$$

- Stage 2 in UKF loop. Time update/prediction steps

$$
\zeta_{i, k \mid k-1}^{j}=f_{j}\left(\mathbf{X}_{i, k-1}^{j}\right), i=0, \ldots, 2 n
$$




$$
\begin{gathered}
\hat{\mathbf{x}}_{k \mid k-1}^{j}=\sum_{i=0}^{2 n} W_{i}^{(m)} \zeta_{i, k \mid k-1}^{j} \\
\mathbf{P}_{k \mid k-1}^{j}=\sum_{i=0}^{2 n} W_{i}^{(c)}\left[\zeta_{i, k \mid k-1}^{j}-\hat{\mathbf{x}}_{k \mid k-1}^{j}\right]\left[\zeta_{i, k \mid k-1}^{j}-\hat{\mathbf{x}}_{k \mid k-1}^{j}\right]^{T}+\mathbf{Q}_{k-1}^{j} \\
\mathbf{Z}_{i, k \mid k-1}^{j}=h\left(\zeta_{i, k \mid k-1}^{j}\right) \\
\hat{\mathbf{z}}_{k \mid k-1}^{j}=\sum_{i=0}^{2 n} W_{i}^{(m)} \mathbf{Z}_{i, k \mid k-1}^{j}
\end{gathered}
$$

- Stage 3 in UKF loop. Measurement update/ correction steps

$$
\begin{gathered}
\mathbf{P}_{z z}^{j}=\sum_{i=0}^{2 n} W_{i}^{(c)}\left[\mathbf{Z}_{i, k \mid k-1}^{j}-\hat{\mathbf{z}}_{k \mid k-1}^{j}\right]\left[\mathbf{Z}_{i, k \mid k-1}^{j}-\hat{\mathbf{z}}_{k \mid k-1}^{j}\right]^{T}+\mathbf{R}_{k}^{j} \\
\mathbf{P}_{x z}^{j}=\sum_{i=0}^{2 n} W_{i}^{(c)}\left[\boldsymbol{\zeta}_{i, k \mid k-1}^{j}-\hat{\mathbf{x}}_{k \mid k-1}^{j}\right]\left[\mathbf{Z}_{i, k \mid k-1}^{j}-\hat{\mathbf{Z}}_{k \mid k-1}^{j}\right]^{T} \\
\mathbf{K}_{k}^{j}=\mathbf{P}_{x z}^{j}\left(\mathbf{P}_{z z}^{j}\right)^{-1} \\
\hat{\mathbf{x}}_{k \mid k}^{j}=\hat{\mathbf{x}}_{k \mid k-1}^{j}+\mathbf{K}_{k}^{j} \mathbf{v}_{k}^{j}
\end{gathered}
$$

where

$$
\begin{gathered}
\mathbf{v}_{k}^{j}=\mathbf{z}_{k}-\hat{\mathbf{z}}_{k \mid k-1}^{j} \\
\mathbf{P}_{k \mid k}^{j}=\mathbf{P}_{k \mid k-1}^{j}-\mathbf{K}_{k}^{j} \mathbf{P}_{z z}^{j} \mathbf{K}_{k}^{j T}
\end{gathered}
$$

The samples are propagated through true nonlinear equations so that the linearization is unnecessary and calculation of the Jacobian matrix is not required.

\subsection{Model probabilities update}

According to the model likelihood and model transition probability governed by the finite-state Markov chain, the model probability $\boldsymbol{\mu}_{k}^{j}$ is updated:

$$
\boldsymbol{\mu}_{k}^{j}=\frac{1}{c} \Lambda_{k}^{j} \overline{\mathbf{c}}_{j}
$$

where

$$
c=\sum_{j=1}^{r} \overline{\mathbf{c}}_{j} \Lambda_{k}^{j}
$$

and

$$
\Lambda_{k}^{j}=\frac{1}{\sqrt{2 \pi\left|\mathbf{P}_{z z}^{j}\right|}} \exp \left[-\frac{1}{2} \mathbf{v}_{k}^{j T}\left(\mathbf{P}_{z z}^{j}\right)^{-1} \mathbf{v}_{k}^{j}\right]
$$

\subsection{Combination of model-individual estimates and covariances}

The model-individual estimates and covariances are combined to overall estimates of state and covariance.

$$
\hat{\mathbf{x}}_{k \mid k}=\sum_{j=1}^{r} \hat{\mathbf{x}}_{k \mid k}^{j} \boldsymbol{\mu}_{k}^{j}
$$

$$
\mathbf{P}_{k \mid k}=\sum_{j=1}^{r} \boldsymbol{\mu}_{k}^{j}\left\{\mathbf{P}_{k \mid k}^{j}+\left[\hat{\mathbf{x}}_{k \mid k}^{j}-\hat{\mathbf{x}}_{k \mid k}\right]\left[\hat{\mathbf{x}}_{k \mid k}^{j}-\hat{\mathbf{x}}_{k \mid k}\right]^{T}\right\}
$$

\section{Fuzzy adaptive filter strategy}

Fig. 1 shows the flow chart of the proposed fuzzyIMMUKF implementation. The two blocks indicated by the dashed-line are the fuzzy logic adaptive systems for determining the value of process noise covariance. The rest represents the IMMUKF loop.

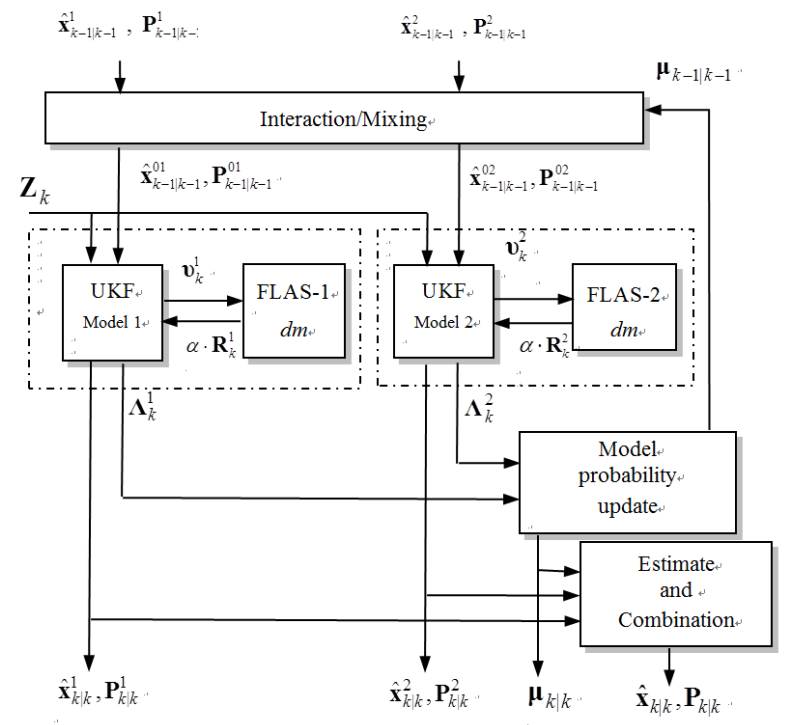

Fig. 1. Flow chart of the Fuzzy-IMMUKF.

\subsection{The fuzzy logic adaptive system (FLAS)}

Initially proposed by L.A. Zadeh in 1965, the fuzzy set theory provides a logical system to deal with the logic of human reasoning process, and can be used to design an intelligent system to resolve semantic analysis or descriptive language. As a result, the fuzzy system can be defined as using fuzzy set theory and fuzzy logic, and combined with the traditional control theory. That is especially useful for applying to a system where a mathematical model is difficult to establish.

A fuzzy system is used to detect the divergence and adapt the filter parameters of UKF. Mamdani proposed a fuzzy modeling approach to model nonlinear systems. The output is the weighted mean of the y:

$$
y=\frac{\sum_{i=1}^{u} u_{y}\left(w_{i}\right) w_{i}}{\sum_{i=1}^{u} w_{i}}
$$

where $u$ represents the membership function, weights $w_{i}$ is calculated by norm operator. The basic structure of the fuzzy system is shown in Fig. 2 


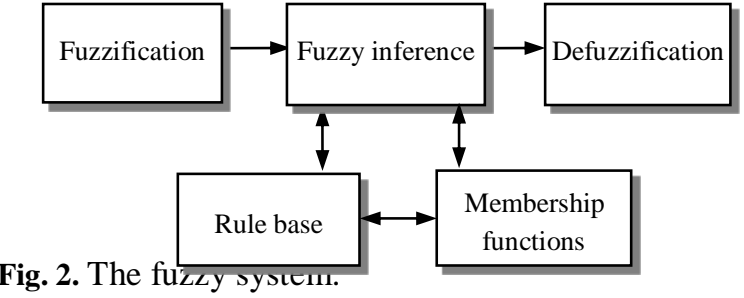

\subsection{The fuzzy interacting multiple model unscented Kalman filter}

A covariation matching technology is employed as the basis for adaption. The design of fuzzy inference system for real-time tuning of the measurement noise covariance is adopted according to the ratio of innovation covariance and the theoretical covariance, hence the accuracy and reliability of the filter are improved. follows:

The calculation of UKF gain is rewritten as

$$
\mathbf{K}_{k}=\mathbf{P}_{x z}\left(\mathbf{P}_{z z}\right)^{-1}
$$

where

$$
\mathbf{P}_{z z}=\sum_{i=0}^{2 n} W_{i}^{(c)}\left[\mathbf{Z}_{i, k \mid k-1}-\hat{\mathbf{z}}_{k \mid k-1}\right]\left[\mathbf{Z}_{i, k \mid k-1}-\hat{\mathbf{z}}_{k \mid k-1}\right]^{T}+\mathbf{R}_{k}^{\prime}
$$

The parameter referred to as the degree of matching ( $d m$ ) is defined as follows:

$$
d m=\frac{\operatorname{tr}\left(C_{\mathbf{v}_{k}}\right)}{\operatorname{tr}\left(\mathbf{P}_{z z}\right)}
$$

The innovation covariance matrix with length $\mathrm{N}$ is given by:

$$
C_{\mathbf{v}_{k}}=\frac{1}{N} \sum_{j=j_{0}}^{k} \mathbf{v}_{j} \mathbf{v}_{j}^{T}
$$

The predicted residual is defined as the difference between the observed measurement of the filter and the predicted one, and is given by $\mathbf{v}_{k}^{j}=\mathbf{z}_{k}-\hat{\mathbf{z}}_{k \mid k-1}^{j}$. In order to tune $\mathbf{R}_{k}$, the parameter $\mathbf{R}_{k}^{\prime}=\xi \cdot \mathbf{R}_{k}$ is defined, and $\xi$ is the measurement noise tunning factor. According to the above analysis, when $\mathbf{R}_{k}$ is appropriately tuned, $d m$ sholud approach 1 . If the strength of the measurement noise increases, $d m$ should increase. For such case, $\mathbf{R}_{k}$ should be enlarged so as to bring $d m$ back to 1 . On the contrary, in the case that measurement noise decreases, $d m$ should also decrease and $\mathbf{R}_{k}$ should be reduced so as to bring $d m$ back to 1 . When $\xi=1$, it becomes the ordinary measurement noise covariance.

The input of the fuzzy controller is $d m$ and the output is $\xi$. There are three fuzzy sets for the input, namely normal large $\cdot$ small. The Sugeno type fuzzy logic system is employed, where the If-Then form consists of three rules for each of the two FLAS systems. For the FLAS in Model 1
1. If $d m$ is small Then $\xi=d m+0.3$

2. If $d m$ is normal Then $\xi=1$

3. If $d m$ is large Then $\xi=2 * d m+5$

For the FLAS in Model 2

1. If $d m$ is small Then $\xi=d m+0.5$

2. If $d m$ is normal Then $\xi=1$

3. If $d m$ is large Then $\xi=2 * d m+20$

The membership functions are shown in Fig. 3

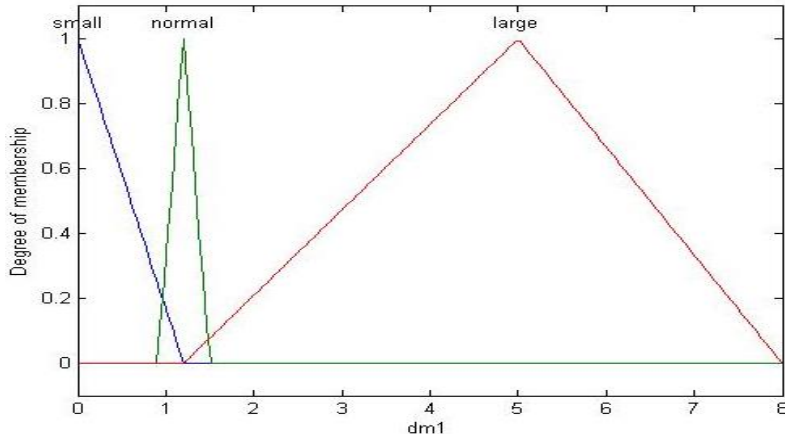

(a) Model 1

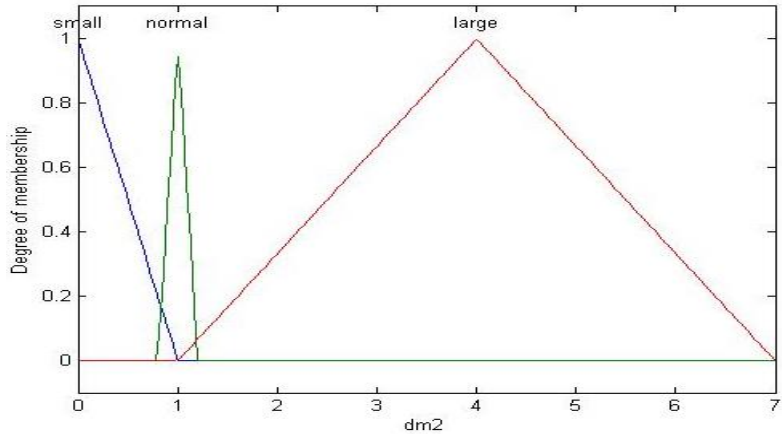

(b) Model 2

Fig. 3. Membership functions of the output fuzzy variable $\xi$ for the two models in the IMM.

\section{Simulation experiments and analysis}

Simulation experiments have been carried out to evaluate the performance of the proposed approach in comparison with the conventional methods for GPS navigation processing. To validate the effectiveness of the proposed approach in time varying satellite signal quality, simulation is conducted for tuning the measurement noise covariance. The commercial software Satellite Navigation (SATNAV) Toolbox by GPSoft LLC was employed for generating the satellite positions and pseudoranges. The satellite constellation was simulated and the error sources corrupting GPS measurements include ionospheric delay, tropospheric delay, receiver noise and multipath. Assume that the differential GPS mode is used and most of the errors can be corrected, but the multipath and receiver thermal noise cannot be eliminated. The multipath interfences are added into the GPS pseudorange observation data during the vehicle moving. For testing the performance due to outlier type multipath interference, there are totally 5 durations where additional randomly generated bias 
errors are intentionally injected onto. Since the research focus on the mitigation of outlier type multipath errors, the influence of measurement noise $\mathbf{R}_{k}$ is relatively critical. A vehicle is designed to perform the uniform motion to reduce the impact on the $\mathbf{Q}_{k}$ caused by unmodelling system dynamic errors. The test trajectory for the simulated vehicle is shown as in Fig. 4. In the simulation, there are 8 GPS satellites available. The skyplot during the simulation time interval is shown as in Fig. 5.

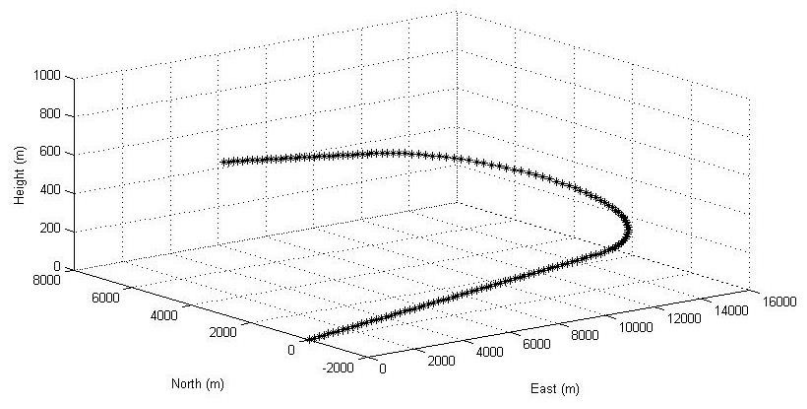

Fig. 4. Test trajectory for the simulated vehicle.

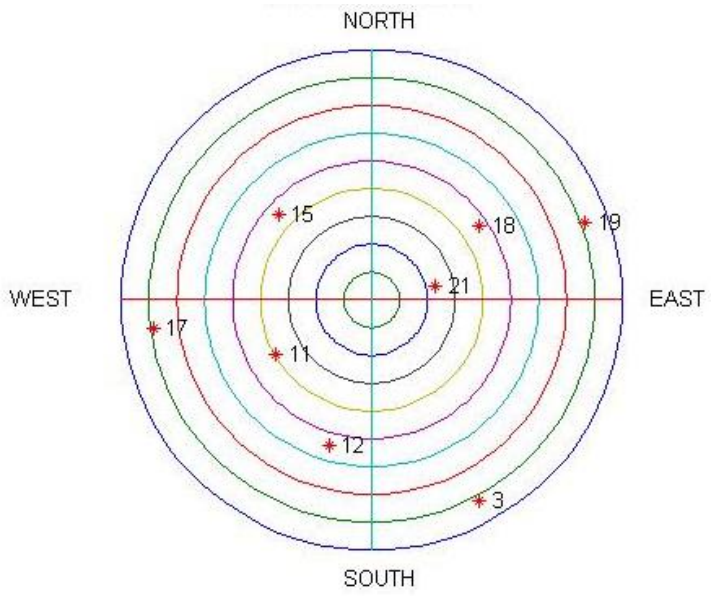

Fig. 5. The skyplot in the simulation.

\subsection{Comparison for UKF and IMMUKF}

GPS navigation accuracy comparsion for UKF and IMMUKF is shown in Fig. 6. Fig. 7 provides the model probabilty based on the IMMUKF method. The uncertainty of the noise can be described by a set of switching models, this modelling approach makes it possible to employ the multiple model estimation to deal with the problem of nonlinear filtering with noise uncertainty. The interaction multiple models used in this paper is employed for dealing with the measurement uncertainty by tuning the tuning $\mathbf{R}_{k}$.

Although it can be seen that the IMMUKF does provide improved accuracy than the UKF, however, it doesn't mitigate the multipath error remarkably.

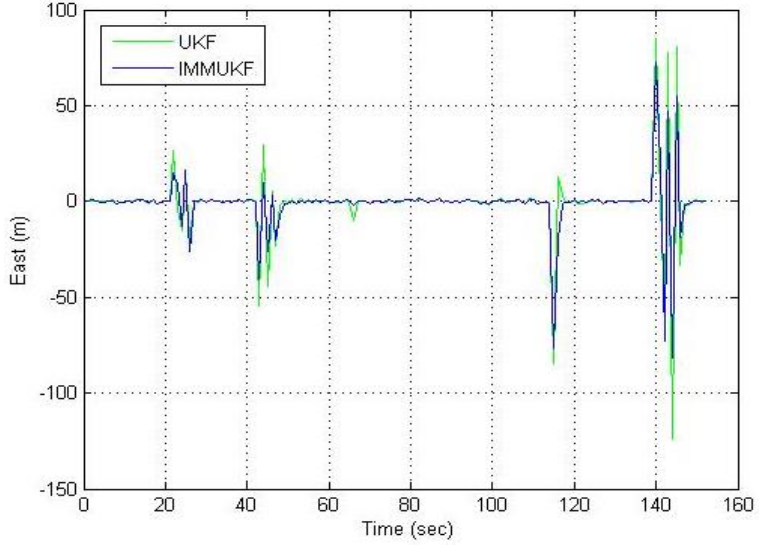

(a) East

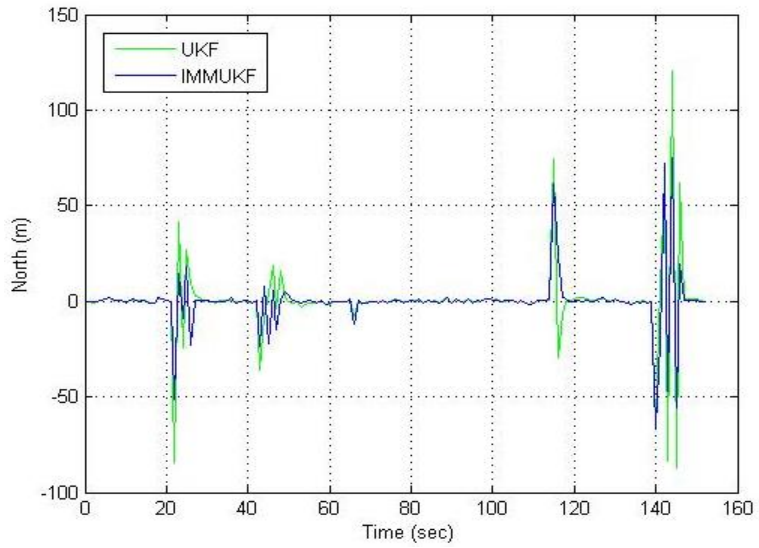

(b) North

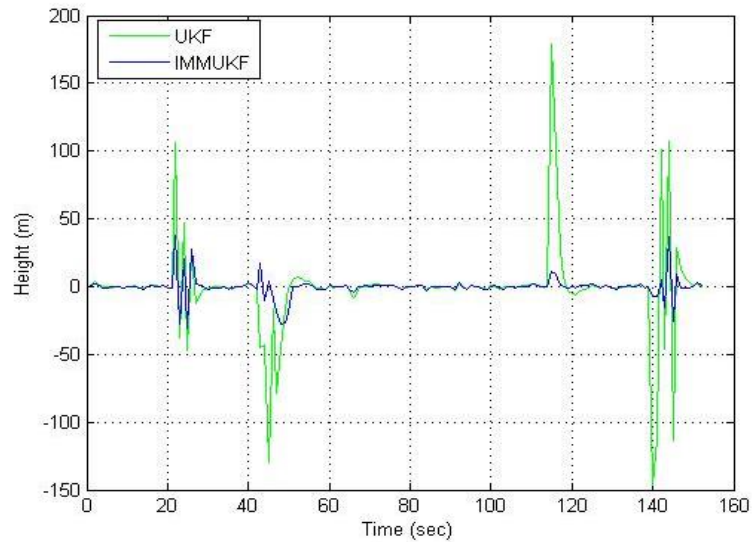

(c) Height

Fig. 6. Navigation accuracy comparsion for UKF and IMMUKF.

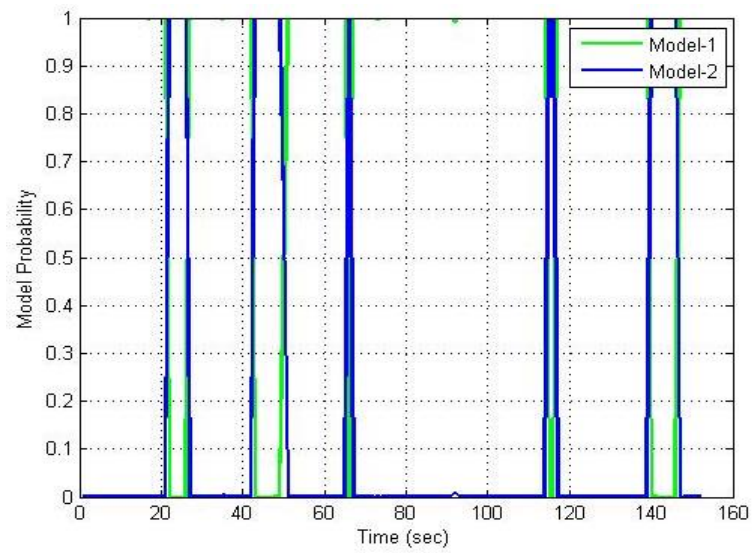

Fig. 7. Model probabilty of IMMUKF. 


\subsection{Further performance enhancement using Fuzzy-IMMUKF}

Utilization of the fuzzy-IMMUKF is the other treatment for further performance enhancement. Positioning accuracy comparsion for IMMUKF and Fuzzy-IMMUKF is shown in Fig. 8. Fig. 9 shows the model probabilty of IMMUKF. For enhancing further system performance, the fuzzy logic system is introduced to timely adjust the measurement noise covariance according to the innovation. The use of IMM with FLAS enables tuning of an appropriate value for the measurement noise covariance so as to maintain good estimation accuracy. As can be seen form Fig. 8, the fuzzy-IMMUKF provides remarkable improvement as compared to the IMMUKF. Fig. 10 shows the error comparison of the three types of filters, while Fig. 11 shows comparsion of position RMS errors in meters for the three approaches.

\section{Conclusions}

This paper has presented a fuzzy logic based interacting multiple model unscented Kalman filter for GPS navigation processing for the outlier types of multipath errors. Multipath mitigation is crucial for improvement of the positioning accuracy. The conventional unscented Kalman filter does not possess the capability to monitor the change of parameters due to changes in measurement model. An interacting multiplemodel based method is suggested to improve the unscented Kalman filter for navigation processing. The resulting IMMUKF exhibits superior navigation accuracy when compared with the classical UKF algorithm. The fuzzy system can be employed to enhance the IMMUKF performance. The fuzzy system is employed for dynamically adjusting the measurement noise covariance, which will be used in each of the parallel filters in the IMM architecture by monitoring the innovation information so as to provide further improvement in estimation accuracy. By monitoring the degree of matching between the estimated innovation covariance and the theoretical one such that the measurement noise covariance matrix is tuned in real time. These performance comparisons of UKF, IMMUKF and Fuzzy-IMMUKF have been carried out and the proposed Fuzzy-IMMUKF algorithm has demonstrated very promising results in navigational accuracy improvement.

\section{Acknowledgements}

Funding for this work was provided by the Ministry of Science and Technology of the Republic of China under grant number MOST 107-2221-E-019-025.

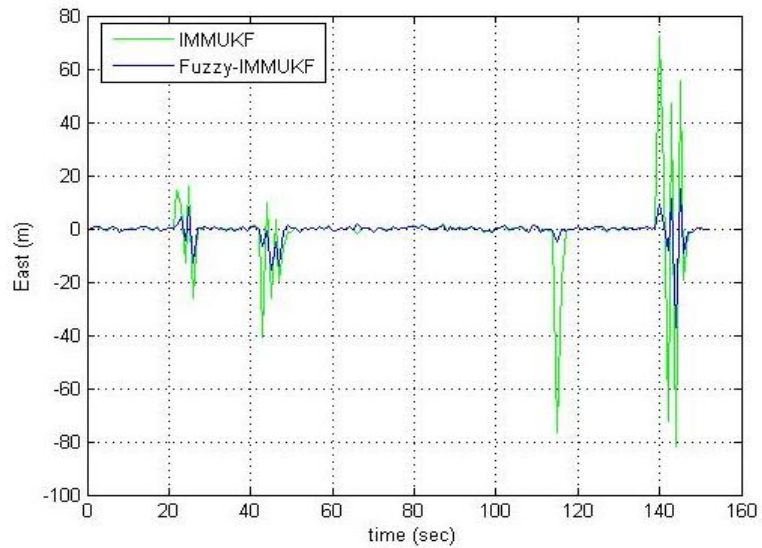

(a) East

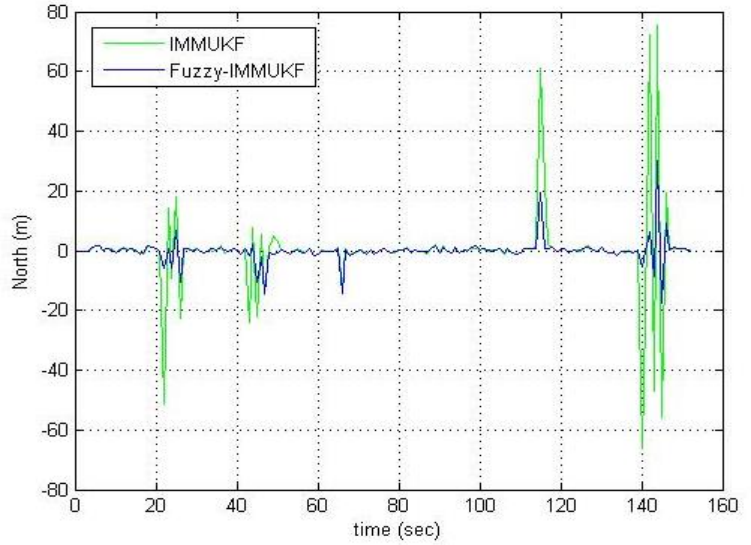

(b) North

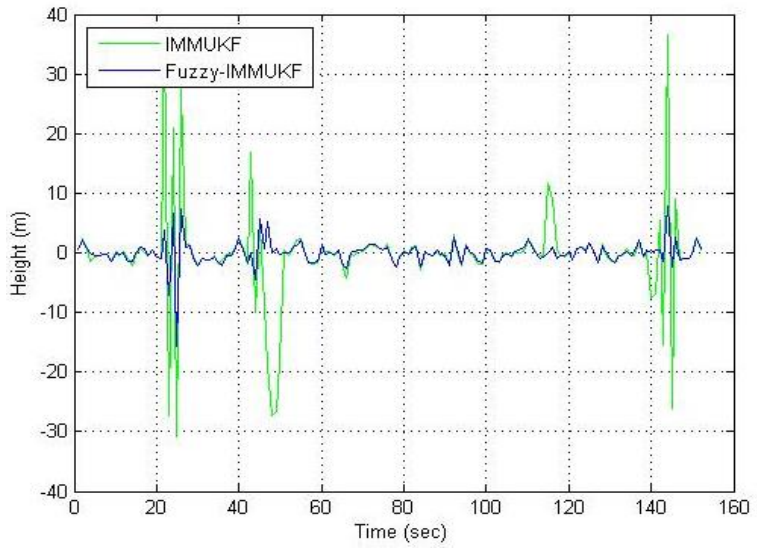

(c) Height

Fig. 8. Positioning accuracy comparsion for IMMUKF and fuzzy-IMMUKF. 


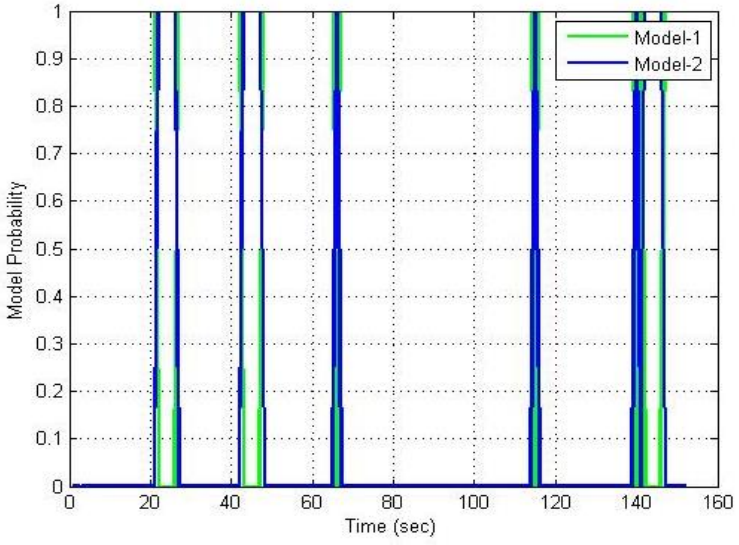

Fig. 9. Model probabilty of the fuzzy-IMMUKF.

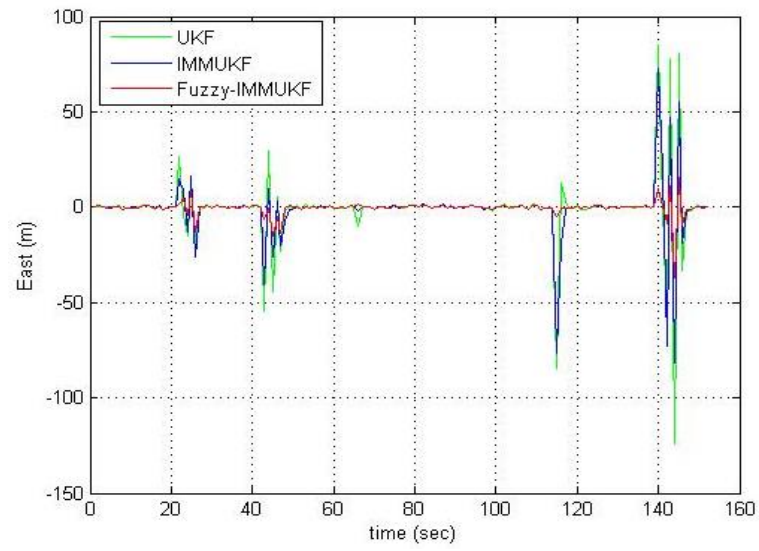

(a) East

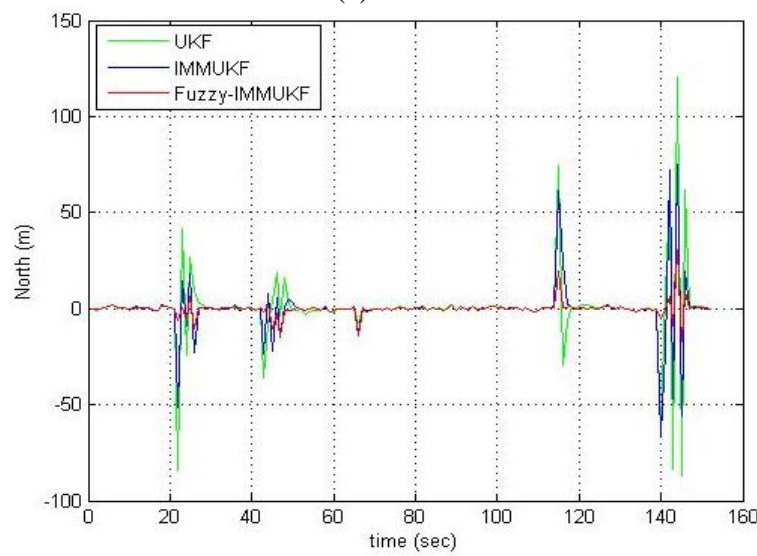

(b) North

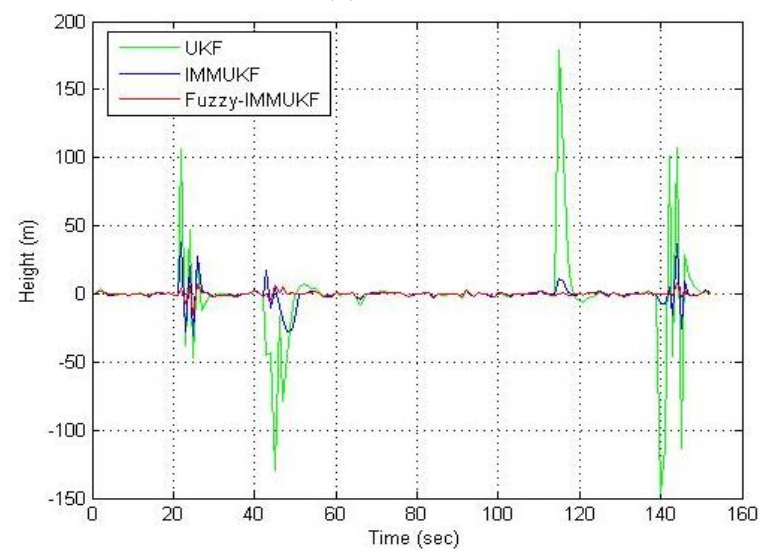

(c) Height

Fig. 10. Positioning accuracy comparsion for UKF, IMMUKF and Fuzzy-IMMUKF.

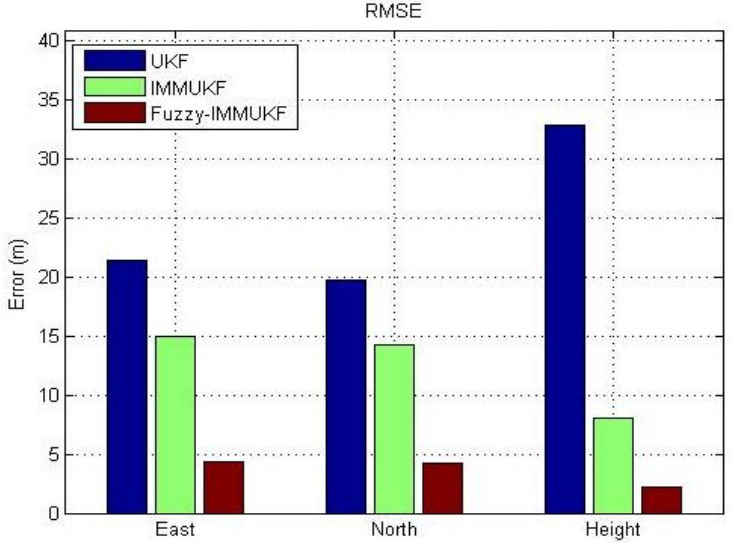

Fig. 11. Comparsion of position accuracies for the three approaches (RMS errors, in meters)

\section{References}

1. L. Cheng, H.Yue, G.Xie, M. F. Ren, Multipath Estimation Using an Intelligent Optimization Algorithm with Non-Gaussian Noise, Proceedings of the 23rd International Conference on Automation \& Computing, UK (2017).

2. N. Sunderhauf, M. Obst, G. Wanielik and P. Protzel, Multipath Mitigation in GNSS-based Localization using Robust Optimization, Proc. of IEEE Intelligent Vehicles Symposium, 2012.

3. N.-S. Zheng, L.-S. Cai, H.-F. Bian, C. Lin, Hybrid particle filtering algorithm for GPS multipath mitigation, Transactions of Nonferrous Metals Society of China, Volume 24, Issue 5, May 2014, pp. 15541561.

4. S. Zair, S. L. Hegarat-Mascle and E. Seignez, Outlier Detection in GNSS Pseudo-Range/Doppler Measurements for Robust Localization, Sensors, 16, 580, 22 pages (2016).

5. A. Pirsiavash, A. Broumandan and G. Lachapelle, Characterization of Signal Quality Monitoring Techniques for Multipath Detection in GNSS Applications, Sensors, 2017, 17, 1579, 24 pages.

6. B. W. Parkinson, J. Spilker, P. Axelrad and P. Enge, Global Positioning System: Theory and Applications, Am. Inst. Aeronaut. Astronaut., Washington D. C., (1996)

7. E. A. Wan R. Van der Merwe, The unscented Kalman filter for nonlinear estimation, In Proc. of IEEE Symposium 2000 (AS-SPCC) (Lake Louise, Oct 2000).

8. D. J. Jwo and C. N. Lai, Unscented Kalman filter with nonlinear dynamic process modeling for GPS navigation, GPS Solutions, 12, pp. 249-260 (2008).

9. H. X. Nie , J. Guan , H. W. Bian, Fuzzy Adaptive Kalman Filtering in INS/ GPS Integrated Navigation System, Ship Electronic Engineering,Vol. 31 No.167 (2011)

10. A. H. Mohamed, K. P. Schwarz, Adaptive Kalman filtering for INS/GPS, Journal of Geodesy, 73, 193-203 (1999)

11. G. Chen and M. Harigae, Using IMM Adaptive Estimator in GPS Positioning, SICE, pp. 25-27 (2001). 
12. Y. Chong, K. Zhang and M. Lu, Exploring a Better IMM-UKF Fusion Algorithm Based on Current Statistical Model in Target Tracking, Journal of Northwestern Polytechnical University, Vol.29, No.6, December (2011).

13. R. C. Zang, P. Y. Cui , H. T. Cui ,Y. Jin, Integrated navigation algorithm based on IMM-UKF, Control Theory \& Applications, Vol. 24, No.4 (2007)

14. D. J. Jwo, F. C. Chung, K. L. Yu, GPS/INS Integration Accuracy Enhancement Using the Interacting Multiple Model Nonlinear Filters, Journal of Applied Research and Technology, Vol. 11, , pp.496-509 (2013)

15. C. H. Tseng, C.-W. Chang and D. J. Jwo, Fuzzy Adaptive Multiple Model Using Nonlinear Filter for Integrated Navigation Sensor Fusion, Sensors, 11, 2090-2111 (2011) 